have not yet been made public. The xperts are scored on their performance Vaslidasion is achieved by quessions the statistical accuracy of an expert's assessments together with how much information they provide. These two quantitative measures of performance are used to calculate performancebased weights.

\title{
Structured expert
} judgment using the Classical Method

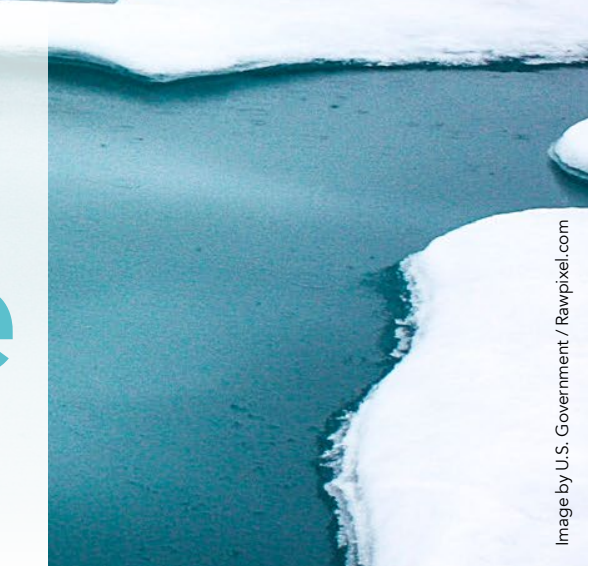

When complex decisions must be made while data is unavailable, structured expert judgment can be used to combine uncertainty distributions resulting from experts' assessments. Roger Cooke, the Chauncey Starr Senior Fellow at Resources for the Future, and Emeritus Professor at Delf University of Technology, created the well-known Classical Model to quantify uncertainty using expert opinion. Together with Dr Tina Nane, from Delt University of Technology, and Dr Anca Hanea, from thert judgment.

- xpert opinions are frequently must be made in situations where ppropriate information cannot be acquired from existing data and models. Experts are asked to quantify their hering unthermo, the experts are unlikely to be in complete agreement with one another. In such situations, expert judgement can be employed to quantify the uncertainty that ensues and to ggregate expert opinion.

Roger Cooke, the Chauncey Starr Senior Fellow at Resources for the Future, and Emeritus Professor at Delf University of Technology, created the Classical Model, also known as Cooke method, for quantifying uncertainty when using expert opinion. Throughout the three decades since its formulation, the Classical Model has been used to perform structured expert judgment in a diverse range of applications, including epidemiology, public and global health, ecology, aviation, nuclear safe environment, and ecology. In addition together with Dr Tina Nane, also from Deff University or Technology, and DrAnca Hanea, from the University of Melbor structured expert judgment. Expert judgment can range from asking
an individual expert for their best guess, to following a formal, structured appro to systematically obtain and combine probabilistic judgments. This synthesis of opinions is called expert elicitation. The validation of expert judgments is challenging since they are only called for when other data are unavailable. Measuring their accuracy is, therefore, an arduous task.

STRUCTURED EXPERT JUDGMENT Structured expert judgment aggregates experts' uncertainty distributions. jooke explains that structured expert 'quantify uncertainty, not to remove it 'from the decision process'. If expert data is to be recognised as scientific data, it should be subjected to the same quality controls as any or thind of data. He proposed a class of methods, known sastify four principles requed, method described as 'scientific'. These are scrutability/accountability, neutrality, fairness, and empirical quality control. Cooke's Classical Model is arguably the most rigorous method for quantifying uncertainty by using expert opinion.

THE CLASSICAL MODEL The naming of the 'Classical Model' highlights the methods association with classical statistics. The Classical Model uses objective performance measures to validate expert opinion. The experts assess uncertain target questions together with a set of calibration questions. The calibration questions are from the experts' field of knowledge, involve dat from official reports
The Classical Model combines experts' distributions using these performance-based weights, optimising the performance of the combined expent, or 'Decision Maker'. Several trong mathematical issues arise Cole aggregation. In non-technical terms, measure must rew the pefformance stastistical accuracy and informativeness, while discouraging the experts from stating judgments tha exffer from the true opinions. The performance of the Decision Maker can be evaluated in the same way as that of the experts using the same performance measures. Performance-optimised Decision Makers correspond to virtual experts and can be adopted by the real-life decision maker. Cross validation is also applied whereby subsets of calibration variables are used to form weights and predict the excluded calibration variables.

APPLICATIONS

Numerous studies have been conducted using the Classical Model. Highlights among these include its application to being caried out by the Europeanch Union and the United States Nuclear Regulatory Commission. During the prolonged volcanic eruption on the ista of Montserrat in the West Indies, from 1995 to 2018, the Classical Model was key decision-support procedure. Hanard University and the Kuwait government used Cooke's method in their 2004-2005 study of fine particulates, pollution in the form of tiny particles or droplets suspended in the air. It was also used in an investigation into foodborne diseases or the World Health Organization in 2011-2013.

LLNESSES TRANSIMITTED BY FOOD AND WATER ore recently, the Centers for Disease Control and Prevention and the University of Florida, together with Roger Cooke, Tha Nane, and Willy Aspinall, perform a large, struct work to control and preventillnesses transmitted through food and water in the United States. The expert elicitation took place at a two-day workshop in May 2017 and involved 48 experts from various professional and scientific backgrounds. Estimates were obtained for the proportion of 33 pathogens including bacteria, such as Salmonella and Legionella, and viruses, such as norovirus and hepatitis $A$, attributed to each of five major transmission pathways (foodborn waterborne, person-to-person, animal and six associated sub-pathways.

The researchers commented on how the method made it possible for the estimates

fter the fact by adding qualifiers such as 'highly confident', 'most likely', satiffactory when din. This may be to take an umbrella to work, but not when deciding how society should deal with climate issues impacting life as we know it The uncritical agregation of high confidences sets and baits the 'confidence trap': thinking that high confidence in each of several statements confers high confidence in all statements jointly. Consider: you may be highly confident that a six will not come up on the first throw of a dice, and on the second, and third and fourth. However, the probability is about one half that a six will come up on one of the four throws. Reasoning under uncertainty must obey the laws of probability, even if the probabiths

Structured expert judgment methods are intended to "quantify uncertainty, not to

remove it from the decision process'.

to be informed by multiple data sources, such as outbreak survellance data, studies of sporadic ilhesses, case reports, and the experts' professional knowledge. They also pointed out that using calibration questions to weigh expert responses, a unique fea we the Classical Model, 'with other elicitation meal rigor not fou findings provide an understanding multiple transmission pathways for the identified pathogens and support the targeting of resources and prioritisation of public health interventions, as well as informing policy.

CLIMATE CHANGE

Climate change is riddled with uncertainty, and Cooke observes tha both the scientific community and the general population make errors when reasoning under uncertainty, and fail to convey it accurately. Faced with multip uncertain quantities, most people will identify what they consider the most likely outcome for each quantity and then reason as if those values were uncertainty to the lay public is difficul, but if the communicators themselves well-nigh istand uncertainty, then it is welnigh impossible. This, in Cooke's with clim is ajor challenge of dealing before the facts are in. Thust decide deciding under uncertainty - which we do very badly.

In a review of 49 professionally contracted studies, Cooke highlighted that the use of expert subjective probabilities is scientific. This evaluation revealed pervasive overconfidence how the role of domain expertise and experience can affect statistical accuracy and informativeness. Moreover, the review demonstrated the need for cross validation, to gauge how well performance on calibration variables

Researchers at the University of Bristol, UK, Princeton University and Rutgers the challenges involved in ensuring predicts performance on the variables 
University in the US, and Resources for the Future, have completed a signific into clima the Classical Model to investigate the contribution made by the dynamic effects of ice sheets to the global mean sea-level rise.

Forecasting the imminent rise in sea leve is challenging. Even so, the quantification of future sea-level rise uncertainties, particularly upper-end estimates, are urgently required to inform adaptation strategies. Expert elicitation took place at two separate, two-day workshops held in the US and UK in 2018 and involved 22 experts. The format and questions were identical, so that the findings could be combined using the Classical Method. The research team found hat by 2100 than twice the upper value put forwad by the United Nations Intergovernment Panel on Climate Change in the Fifth Assessment Report. Moreover, this would have profound consequences for humanity with a potential land loss of 1.79 million $\mathrm{km}^{2}$, including areas of food production, and up to 187 million people displaced.

The article detailing this study, published in the Proceedings of the National

Academy of Sciences of the United States of America (PNAS), has received a great deal of attention. It has been mention in 331 news stories from 263 outlets, and as the 70th most-discussed paper in 2019 , it sils in the top $5 \%$ of all reser.

ONLINE MODULE

The Classical Model is also the focus of a Massive Open Online Course (MOOC): 'Decision Making Under Uncertainty: Introduction to Structured Expert Judgment', presented by Roger Cooke, Tina Nane, and Anca Hanea. This course is arranged into six parts, combining theory and applications in an interactive and engaging manner.

Learners are introduced to structured expert judgment methods, particularly the Classical Model, and advised as to why and when they should be applied. They learn how to account for uncertainty assessments and biases

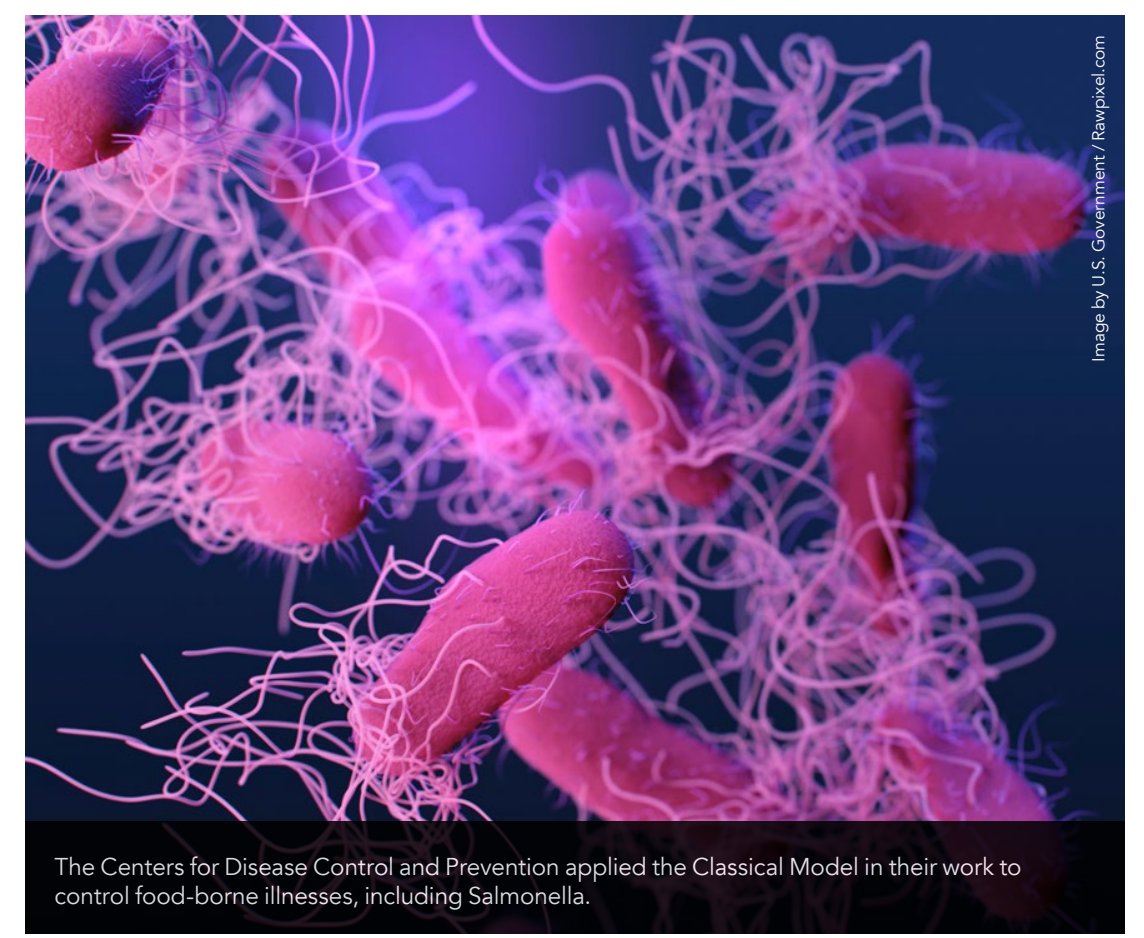

Using calibration to weight expert responses, a unique feature of the Classical Model, 'introduces mathematical rigor not found with other elicitation methods'.

setting. There is also the opportunity to analyse expert data with EXCALIBUR or Andunl, sofware packages obtain answers to questions of interest. Optional modules include exploring dependence elicitation and eliciting probabilities, applying structured expert-judgment methods to realworld scenarios, and using a different method, the IDEA Protocol module. This is the only available module on expert judgment. In its three runs to date, it has attracted more than 7,000 participants from 121 countries

A follow-up online course is dedicated to applying structured expert judgment. Learners have the opportunity to perform their own study, under close guidance from Tina Nane and Roger
Cooke. The learners will design the study and gather, assess the performance of and combine, expert opinion. They will also carry out the analysis and report the
indings of their study.

EVALUATION OF THE

Cooke has collected expert data from many studies over the years to evaluate the Classical Model and compare it with other possible weighting schemes, including equal weighting and quantile aggregation. The Classical Model outperformed the other aggregation methods considered in the analysis. The findings demonstrate the superiority of the Classical Model, both in terms of in-sample and out-of-sample validation and in terms of point forecast accuracy. Moreover, when compared with other aggregation methods, the performanceby the Classical Model is rerts generated accurate and more informative.

\section{Behind the Research}

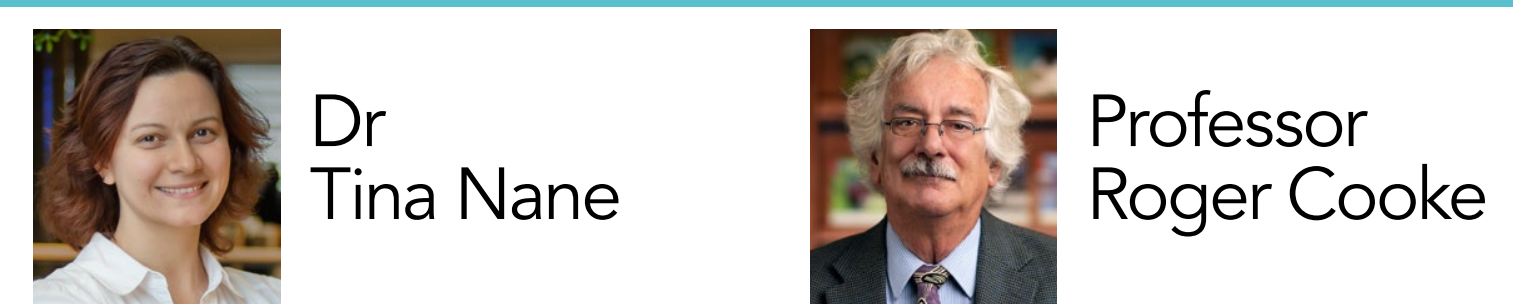

E: g.f.nane@tudelft.n! W: www.tinanane.com E: cooke@rff.org W: rogermcooke.net

Research Objectives

Tina Nane has designed the Massive Open Online Course (MOOC) 'Decision making under uncertainty: an introduction to structured expert judgment', based on Roger Cooke's Classical Model.

\section{Bic}

Tina Nane is an Assistant Professor of Applied Probability at Delft University of Technology. She received her PhD in Statistics in 2013 and a cum laude MSc degree in Risk and Environmental Modelling in 2008 from Delft University of Technology. Dr Nane's current research primarily focuses on uncertainty quantification and analysis, both datadiven and by employing expert opinio Dr Nane has designed and is the main Course (MOOC) 'Decision Making Under

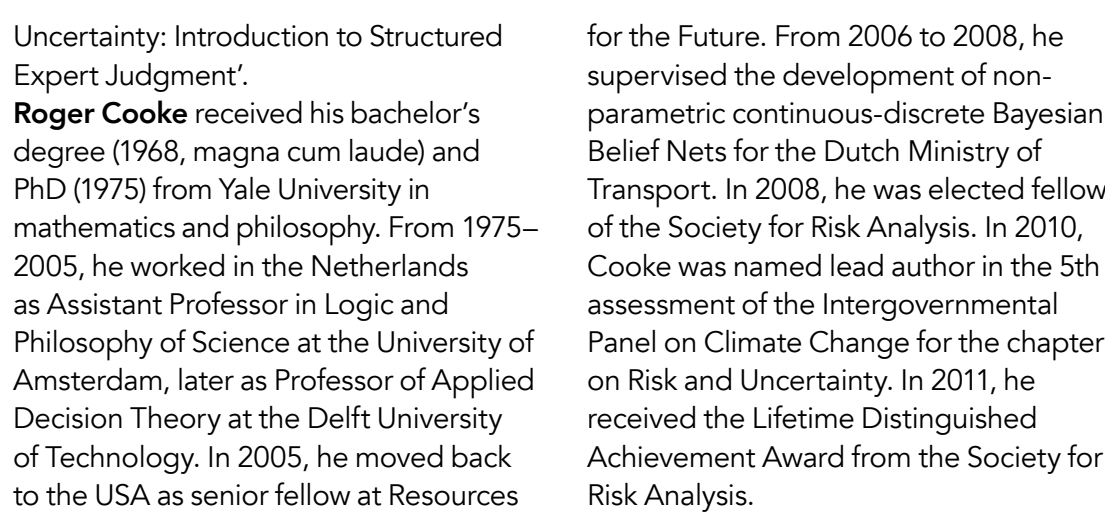
Uncertainty: Introduction to Structured Expert Judgment'

Roger Cooke received his bachelor's degree (1968, magna cum laude) and PhD (1975) from Yale University in mathematics and philosophy. From 19752005 , he worked in the Netherlands as Assistant Professor in Logic and Amsterdam loter as Profese University of Decision Theory at the Delf University of Technology. In 2005, he moved back to the USA as senior fellow at Resources

for the Future. From 2006 to 2008, he supervised the development of nonparametric continuous-discrete Bayesian Belief Nets for the Dutch Ministry of of the Society for Risk Analysis. In 2010, Cooke was named lead author in the 5 th assessment of the Intergovernmental Panel on Climate Change for the chapter on Risk and Uncertainty. In 2011, he Achievement Award from the Society for Risk Analysis.

\section{References}

Decision Making Under Uncertainty:
Introduction to Structured Expert dine-eaning tudelftelcoursect. decision-making-under-uncertaintyIntroduction-to-structured-expert-

Beshearse, E, Bruce, BB, Nane, GF,
Cooke, RM, Aspinall, W, Hald, T, et al, (2021) Attribution of lininesses Transmited by food and
to Comprehensive Transmission Pathways Using Structured Expert

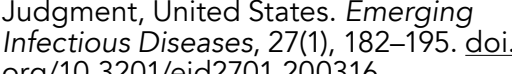
Hanea, AM, Nane, GF, (2021) An In-
Depth Perspective on the Classical Model. In: Hanea, AM, Nane, GF, Judgement in Risk and Decision Analysis. International Series in

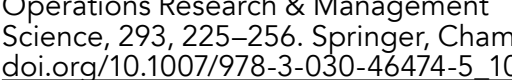
Cooke, R, (2020) The Science of
Forecasting: The Special Sauce. Forecasting: The Special Sauce.
Resources, Ionlinel. www.resources.

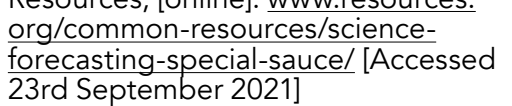

Cooke, RM, Marti, D, Mazzuchi, T,
(2020) Expert forecasting with and without uncertainty quantification
and weighting: what do the data say? International Journal of Forecasting
$37,378-387$. doi.org $/ 10.1016 /$ i. ifforecast.2020.06.007

Bamber, J, Oppenheimer, M, Kopp,
R, Aspinall, W, Cooke, R, (2019) Ice sheet contributions to future sea-level
rise from structured expert judgment. rise from structured expert judg
PNAS, 116(23), 11195-11200. doi.
org/10.1073/pnas.1817205116 org/10.1073/pnas.1817205116

Cooke, R, (2019) The Iceman Cometh.
Resources Ionlinel. www resources or common-resources/iceman-cometh
[Accessed 23rd September 2021]

Cooke, R, (2017) Respectability Looming for Expert Judgment. Resolrces [online]. www.resources. org/common-resources/respectab
looming-for-expert-judgment
[Accessed 23rd September 2021] Cooke, RM, (2015) Messaging climate Change, 5, 8-10. www.nature. com/nclimate/journwal/v5/n1/full/
nclimate2466.html
Personal response

What inspired you to include performance-based weights in the

II Expert subjective probabilities analyses of nuclear power plants in the 1970s. The rigorous reporting in these early studies exposed very wide differences in experts valgments and teed up issues of judgment. Any new measurement device, such as Galileo's telescope, is first 'calibrated' by applying it to Expert judgment constitunt know. measuring device. Applying this simple idea led to treating experts as these hypotheses against calibration variables from their field to which the
true values were known.

\section{TंUDelft} things we know before employing statistical hypotheses and validating 